International Mathematical Forum, Vol. 8, 2013, no. 32, 1569 - 1576

HIKARI Ltd, www.m-hikari.com

http://dx.doi.org/10.12988/imf.2013.38150

\title{
Right Derivations on Semirings
}

\author{
S. P. Nirmala Devi \\ Department of Mathematics \\ Sree Sowdambika College of Engineering \\ Aruppukottai - 626101, India \\ spnirmala1980@gmail.com \\ M. Chandramouleeswaran \\ Department of Mathematics \\ Saiva Bhanu Kshatriya College \\ Aruppukottai- 626101, India \\ moulee59@gmail.com
}

Copyright (C) 2013 S. P. Nirmala Devi and M. Chandramouleeswaran. This is an open access article distributed under the Creative Commons Attribution License, which permits unrestricted use, distribution, and reproduction in any medium, provided the original work is properly cited.

\begin{abstract}
Motivated by some works on derivations on rings, Chandramoulesswaran and Thiruveni discussed the notion of derivations on semirings. In this paper, we discuss the notion of right derivations on semirings and prove some simple properties.
\end{abstract}

Mathematics Subject Classification: 16Y60

Keywords: Semirings, Derivations, right-derivations

\section{Introduction}

Richard Dedekind used the notion of semiring in his study of ideals without giving the semiring a formal definition. However, H.S. Vandiver, [5] gave a formal definition and introduced the notion of semiring in 1934. A natural 
example of a semiring which is not a ring is the set of natural numbers in $\mathbb{N}$ under usual addition and multiplication of numbers.

Even though, the study of derivations in rings was initiated long back, it got its significance only after Posner [4] who in 1955 established two very striking results. The notion of derivation has also been generalized in various directions. In [3], Jonathan Golan mentioned about the derivation on a semiring. However, nothing more has been said on it. This motived Chandramouleeswaran and Thiruveni to introduce and discuss the notion of derivation on a semiring and its properties[2]. In 1990, Bresar and Vukman [1] firstly introduced the notion of a left- derivation in a ring and proved that a left- derivation of a semiprime ring $R$ must map $R$ into its center. Motivated by this, in this paper, we introduce the notion of right-derivation on a semiring $S$ and prove some simple properties.

\section{Preliminaries}

In this section we recall some basic definitions on semirings and derivations on it, that are needed for our work.

Definition 2.1 A semiring $(S,+, \cdot)$ is an algebraic system with a nonempty set $S$ together with two binary operations + and $\cdot$ such that

1. $(S,+)$ is a semi group

2. $(S, \cdot)$ is a semi group

3. For all $a, b, c \in S, a \cdot(b+c)=a \cdot b+a \cdot c$ and $(a+b) \cdot c=a \cdot c+b \cdot c$ hold.

Definition 2.2 A semiring $(S,+, \cdot)$ is said to be additively commutative if $(S,+)$ is a commutative semi group. A semiring $(S,+, \cdot)$ is said to be multiplicatively commutative if $(S, \cdot)$ is a commutative semi group. It is said to be commutative if both $(S,+)$ and $(S, \cdot)$ are commutative.

Definition 2.3 The semiring $(S,+, \cdot)$ is said to be a semiring with zero, if it has an element 0 in $S$ such that $x+0=x=0+x$ and $x \cdot 0=0=0 \cdot x \quad \forall x \in S$.

Definition 2.4 A semiring $(S,+, \cdot)$ is said to be a semiring with an identity element 1 , if there exists an element $1 \neq 0 \in S$ such that $1 \cdot x=x=x \cdot 1 \forall x \in S$.

Definition 2.5 Let $(S,+, \cdot)$ be a semiring. An element $\alpha$ of $S$ is called additively left cancellative if for all $\alpha, \beta, \gamma \in S, \alpha+\beta=\alpha+\gamma \Rightarrow \beta=\gamma$. If every element of a semiring $S$ is additively left cancellative, it is called an additively left cancellative semiring. 
Analogously, one can define an additively right cancellative semiring.

Definition 2.6 A semiring $(S,+, \cdot)$ is said to be additively cancellative if it is both additively left and right cancellative.

Definition 2.7 Let $S$ be a semiring. A left $S$-semimodule is a commutative monoid $\left(M,+, 0_{M}\right)$ in which scalar mulitiplication $S \times M \rightarrow M$, denoted by $(s, m) \rightarrow s m$, satisfies the following conditions

1. $\left(s s^{\prime}\right) m=s\left(s^{\prime} m\right)$

2. $s\left(m+m^{\prime}\right)=s m+s m^{\prime}$

3. $\left(s+s^{\prime}\right) m=s m+s^{\prime} m$

4. $1_{S} m=m$

5. $s 0_{M}=0_{M}=0_{S} m \quad \forall s, s^{\prime} \in S \quad \forall m, m^{\prime} \in M$.

If $V(M)=M$ then $M$ is an $S$-module where $V(M)$ is the set of all elements of $M$ having additive inverse.

Definition 2.8 Let $S$ be a semiring.

1. $S$ is said to be prime if $a S b=0 \Rightarrow a=0$ or $b=0$.

2. $S$ is said to be semiprime if $a S a=0 \Rightarrow a=0$.

3. $S$ is said to be 2 torsion free if $2 a=0, a \in S \Rightarrow a=0$.

Definition 2.9 Let $(S,+, \cdot)$ be a semiring and $X$ be $S$ module. A derivation on $S$ is a map $d: S \rightarrow X$ satisfying the following conditions

1. $d(x+y)=d(x)+d(y) \quad \forall x, y \in S$

2. $d(x y)=d(x) y+x d(y), \quad \forall x, y \in S$.

Example 2.10 Let $S$ be a semiring. Let $M_{2}(S)=\left\{\left(\begin{array}{cc}a & 0 \\ b & c\end{array}\right) \mid a, b, c \in S\right.$. $\}$ Define $d: M_{2}(S) \rightarrow M_{2}(S)$ is given by $d\left[\left(\begin{array}{cc}a & 0 \\ b & c\end{array}\right)\right]=\left(\begin{array}{ll}0 & 0 \\ b & 0\end{array}\right)$

Then $d$ is a derivation on $M_{2}(S)$. 


\section{Right-Derivations}

In this section, we introduce the notion of right-(left-)derivations on semirings, to illustrate the concept, discuss examples and prove some simple properties.

Definition 3.1 Let $S$ be a semiring And $X$ be Left-SModule. An additive map $d_{L}: S \rightarrow X$ is said to be a left-derivation if $d_{L}(x y)=x d_{L}(y)+y d_{L}(x) \forall x, y \in$ $S$.

Example 3.2 Let $S$ be commutative semiring with characteristic 4 . Let $M_{2}(S)=\left\{\left(\begin{array}{ll}a & b \\ b & a\end{array}\right) \mid a, b \in S\right\}$

The map $d_{L}: M_{2}(S) \rightarrow M_{2}(S)$ given by

$$
d_{L}\left[\left(\begin{array}{cc}
a & b \\
b & a
\end{array}\right)\right]=\left(\begin{array}{cc}
0 & 2 b \\
0 & 0
\end{array}\right)
$$

is a left-derivation but not a derivation on $M_{2}(S)$.

Analogously we can define a right derivation on $S$ as follows.

Definition 3.3 Let $S$ be a semiring and $X$ be a right-Smodule. An additive $\operatorname{map}_{R}: S \rightarrow X$ is said to be a right-derivation if $d_{R}(x y)=d_{R}(x) y+$ $d_{R}(y) x \quad \forall x, y \in S$.

Example 3.4 Let $S$ be commutative semiring with characteristic 4 . Let $M_{2}(S)=\left\{\left(\begin{array}{ll}a & b \\ b & a\end{array}\right) \mid a, b \in S\right\}$

The map $d_{R}: M_{2}(S) \rightarrow M_{2}(S)$ given by

$$
d_{R}\left[\left(\begin{array}{cc}
a & b \\
b & a
\end{array}\right)\right]=\left(\begin{array}{cc}
0 & 0 \\
2 b & 0
\end{array}\right)
$$

is a right-derivation but not a derivation on $M_{2}(S)$.

One can easily prove the following.

\section{Lemma 3.5}

1. Sum of two left derivations on an additively commutative semiring is again a left derivation.

2. Sum of two right derivations on an additively commutative semiring is again a right derivation.

Lemma 3.6 Let $S$ be a semiring, $X$ be a righr-Smodule and $d_{R}: S \rightarrow X$ be a right-derivation. For any element $a \in S, d_{R}\left(a^{n}\right)=n d_{R}(a) a^{n-1}$. 
Proof: We prove this result by induction hypothesis to prove this result.

Take $n=2$. Then

$d_{R}\left(a^{2}\right)=d_{R}(a \cdot a)=d_{R}(a) a+d_{R}(a) a=2 a d_{R}(a) a$.

Assume that $d_{R}\left(a^{n}\right)=n d_{R}(a) a^{n-1}$. Now,

$$
\begin{aligned}
d_{R}\left(a^{n+1}\right) & =d_{R}\left(a^{n} \cdot a\right) \\
& =d_{R}\left(a^{n}\right) a+d_{R}(a) a^{n} \\
& =\left[n d_{R}(a) a^{n-1}\right] a+d_{R}(a) a^{n} \\
& =n d_{R}(a) a^{n}+d_{R}(a) a^{n} \\
& =(n+1) d_{R}(a) a^{n+1} \\
d_{R}\left(a^{n}\right) & =n d_{R}(a) a^{n-1} \quad \forall n>0 .
\end{aligned}
$$

Analogously we can prove the following.

Lemma 3.7 Let $S$ be a semiring, $X$ be a left-Smodule and $d_{L}: S \rightarrow X$ be a Left- derivation. For any element $a \in S, d_{L}\left(a^{n}\right)=n a^{n-1} d_{L}(a)$.

Theorem 3.8 Let $S$ be a semiring and $X$ be a 2-torsion free right-Smodule. If $d_{R}: S \rightarrow X$ is a right-derivation then $a, b, c \in R$, then

1. $d_{R}(a b+b a)=2 d_{R}(b) a+2 d_{R}(a) b$.

2. $d_{R}(a b a)=d_{R}(b) a^{2}+3 d_{R}(a) b a-d_{R}(a) a b$.

3. $d_{R}(a b c+c b a)=d_{R}(a)(c b+b c)+3 d_{R}(b) c a+3 d_{R}(c) b a-d_{R}(c) a b-d_{R}(b) a c$.

4. $d_{R}(a) a(b a-a b)=d_{R}(a)(a b-b a) a$.

5. $(b a-a b)\left(d_{R}(b a)-d_{R}(a) b-d_{R}(b) a\right)=0$.

\section{Proof:}

$$
\begin{aligned}
d_{R}(a b) & =d_{R}(a) b+d_{R}(b) a \\
d_{R}(a b+b a) & =d_{R}(a b)+d_{R}(b a) \\
& =d_{R}(a) b+d_{R}(b) a+d_{R}(b) a+d_{R}(a) b \\
& =2 d_{R}(a) b+2 d_{R}(b) a \quad \cdots \cdots(1)
\end{aligned}
$$

Replace $b$ by $a b+b a$ in (1),

$$
\begin{aligned}
d_{R}(a(a b+b a)+(a b+b a) a) & =2 d_{R}(a)(a b+b a)+2 d_{R}(a b+b a) a \\
& =2 d_{R}(a) a b+6 d_{R}(a) b a+4 d_{R}(b) a^{2} \cdots \cdots(2) \\
d_{R}\left(a^{2} b+a b a+a b a+b a^{2}\right) & =d_{R}\left(a^{2} b\right)+2 d_{R}(a b a)+d_{R}\left(b a^{2}\right) \\
& =d_{R}\left(a^{2}\right) b+d_{R}(b) a^{2}+2 d_{R}(a b a)+d_{R}(b) a^{2}+d_{R}\left(a^{2}\right) b \\
& =2 d_{R}(b) a^{2}+4 d_{R}(a) a b+2 d_{R}(a b a) \cdots(3)
\end{aligned}
$$


From (2) and (3)

$$
\begin{aligned}
2 d_{R}(b) a^{2}+4 d_{R}(a) a b+2 d_{R}(a b a) & =2 d_{R}(a) a b+6 d_{R}(a) b a+4 d_{R}(b) a^{2} \\
2 d_{R}(a b a) & =2 d_{R}(b) a^{2}-2 d_{R}(a) a b+6 d_{R}(a) b a \\
d_{R}(a b a) & =d_{R}(b) a^{2}+3 d_{R}(a) b a-d_{R}(a) a b .
\end{aligned}
$$

replace $b$ by $c b+b c$ in (1)

$$
\begin{aligned}
d_{R}(a(c b+b c)+(c b+b c) a)= & 2 d_{R}(a)(c b+b c)+2 d_{R}(c b+b c) a \\
= & 2 d_{R}(a) c b+2 d_{R}(a) b c+4 d_{R}(c) b a+4 d_{R}(b) c a \cdots \cdots(4) \\
d_{R}(a c b+a b c+c b a+b c a)= & d_{R}(a c b)+d_{R}(a b c+c b a)+d_{R}(b c a) \\
= & d_{R}(a c) b+d_{R}(b) a c+d_{R}(a b c+c b a)+d_{R}(b c) a+d_{R}(a) b c \\
= & d_{R}(a) c b+d_{R}(c) a b+d_{R}(b) a c+d_{R}(a b c+c b a)+ \\
& d_{R}(b) c a+d_{R}(c) b a+d_{R}(a) b c \quad \cdots \cdots(5)
\end{aligned}
$$

From (4) and (5)

$$
\begin{aligned}
& 2 d_{R}(a) c b+2 d_{R}(a) b c+4 d_{R}(c) b a+4 d_{R}(b) c a \\
&= d_{R}(a) c b+d_{R}(c) a b+d_{R}(b) a c+ \\
& d_{R}(a b c+c b a)+d_{R}(b) c a+d_{R}(c) b a+d_{R}(a) b c \\
& d_{R}(a b c+c b a)= d_{R}(a)(c b+b c)+3 d_{R}(b) c a+3 d_{R}(c) b a-d_{R}(c) a b-d_{R}(b) a c
\end{aligned}
$$

Replace $c$ by $b a$ in (6)

$$
\begin{aligned}
d_{R}(a b b a+b a b a)= & d_{R}(a)(b a b+b b a)+3 d_{R}(b) b a a+ \\
& 3 d_{R}(b a) b a-d_{R}(b a) a b-d_{R}(b) a b a \\
= & d_{R}(a)\left(b a+b^{2} a\right)+3 d_{R}(b) b a^{2}+ \\
& 3 d_{R}(b a) b a-d_{R}(b a) a b-d_{R}(b) a b a \cdots(7) \\
d_{R}\left(a b^{2} a+(b a)^{2}\right)= & d_{R}\left(a b^{2} a\right)+d_{R}(b a)^{2} \\
= & d_{R}\left(a b^{2}\right) a+d_{R}(a) a b^{2}+2 d_{R}(b a) b a \\
= & d_{R}(a) b^{2} a+d_{R}\left(b^{2}\right) a^{2}+d_{R}(a) a b^{2}+2 d_{R}(b a) b a \\
= & d_{R}(a) b^{2} a+2 d_{R}(b) b a^{2}+d_{R}(a) a b^{2}+2 d_{R}(b a) b a
\end{aligned}
$$

From (7) and (8)

$$
\begin{aligned}
d_{R}(a) b a b+d_{R}(a) b^{2} a+ & 3 d_{R}(b) b a^{2}+3 d_{R}(b a) b a-d_{R}(b a) a b-d_{R}(b) a b \\
= & d_{R}(a) b^{2} a+2 d_{R}(b) b a^{2}+d_{R}(a) a b^{2}+2 d_{R}(b a) b a \\
d_{R}(b a) b a b-d_{R}(b a) a b= & d_{R}(b) a b a-d_{R}(a) b a b-d_{R}(a) b^{2} a-3 d_{R}(b) b a^{2}+ \\
& d_{R}(a) b^{2} a+2 d_{R}(b) b a^{2}+d_{R}(a) a b^{2} \\
d_{R}(b a)(b a-a b)= & d_{R}(b)(a b-b a) a+d_{R}(a)(a b-b a) b \ldots . .
\end{aligned}
$$


Replace $b$ by $a+b$ in $(9)$

$$
\begin{aligned}
d_{R}((a+b) a)[(a+b) a-a(a+b)]= & d_{R}(a+b)[a(a+b)-(a+b) a] a+ \\
& d_{R}(a)[a(a+b)-(a+b) a](a+b) \\
d_{R}\left(a^{2}+b a\right)(b a-a b)= & \left(d_{R}(a)+d_{R}(b)\right)(a b-b a) a+d_{R}(a)(a b-b a)(a+b) \\
\left(d_{R}\left(a^{2}\right)+d_{R}(b a)\right)(b a-a b)= & d_{R}(a)(a b-b a) a+d_{R}(b)(a b-b a) a+ \\
& d_{R}(a)(a b-b a) a+d_{R}(a)(a b-b a) b \\
2 d_{R}(a) a(b a-a b)+d_{R}(b)(a b-b a) a+ & d_{R}(a)(a b-b a) b \\
= & 2 d_{R}(a)(a b-b a) a+d_{R}(b)(a b-b a) a+d_{R}(a)(a b-b a) b \text { using }(9) \\
d_{R}(a) a(b a-a b)= & d_{R}(a)(a b-b a) a \cdots(10)
\end{aligned}
$$

Replace $a$ by $a+b$ in $(10) d_{R}(a+b)(a+b)(b(a+b)-(a+b) b)=d_{R}(a+b)((a+$ $b) b+b(a+b))(a+b)$

$$
\begin{gathered}
d_{R}(a+b)(a+b)\left(b a+b^{2}-a b-b^{2}\right)=\left[d_{R}(a)+d_{R}(b)\right]\left(a b+b^{2}-b a-b^{2}\right)(a+b) \\
\left(d_{R}(a)+d_{R}(b)\right)(a+b)(b a-a b)=\left(d_{R}(a)+d_{R}(b)\right)(a b-b a)(a+b) \\
d_{R}(a) a(b a-a b)+d_{R}(a) b(b a-a b)+d_{R}(b) a(b a-a b)+d_{R}(b) b(b a-a b)= \\
d_{R}(a)(a b-b a) a+d_{R}(b a)(b a-a b)+d_{R}(b)(a b-b a) b u s i n g(9) \\
d_{R}(a) b(b a-a b)+d_{R}(b) a(b a-a b)=d_{R}(b a)(b a-a b) \\
(b a-a b)\left[d_{R}(a b)-d_{R}(a) b-d_{R}(b) a\right]=0 .
\end{gathered}
$$

Analogously we can prove the following.

Theorem 3.9 Let $S$ be a semiring and $X$ be a 2-torsion free left $S$-module. If $d_{L}: S \rightarrow X$ is a left-derivation then $a, b, c \in R$, then

1. $d_{L}(a b+b a)=2 a d_{L}(b)+2 b d_{L}(a)$.

2. $d_{L}(a b a)=a 2 d_{L}(b)+3 a b d_{L}(a)-b a d_{L}(a)$.

3. $d_{L}(a b c+c b a)=3 a b d_{L}(c)+3 a c d_{L}(b)+(b c+c b) d_{L}(a)-b a d_{L}(c)-c a d_{L}(b)$.

4. $(a b-b a) a d_{L}(a)=a(b a-a b) d_{L}(a)$.

5. $(a b-b a)\left(d_{L}(a b)-a d_{L}(b)-b d_{L}(a)\right)=0$.

Theorem 3.10 Let $S$ be an additively commutative and cancellative semiring. Let $X$ be a right $S$-module. Let $d_{R}: S \rightarrow X$ be a non zero right-derivation. Suppose that $x S a=0$ with $a \in S, x \in X \Rightarrow a=0$ or $x=0$. Then $S$ is commutative.

Proof: Let $S$ be an additively commutative and cancellative semiring. 
Let $d_{R}: S \rightarrow X$ be a non zero right-derivation on $S$.

$$
\begin{aligned}
d_{R}(a b a) & =d_{R}(a) b a+d_{R}(b a) a \\
& =d_{R}(a) b a+d_{R}(b) a^{2}+d_{R}(a) b a \\
& =2 d_{R}(a) b a+d_{R}(b) a^{2} \ldots \ldots(1) \\
d_{R}(a b a) & =d_{R}(a b) a+d_{R}(a) a b \\
& =d_{R}(a) b a+d_{R}(b) a^{2}+d_{R}(a) a b \\
& =d_{R}(a)(b a+a b)+d_{R}(b) a^{2} \ldots \ldots
\end{aligned}
$$

From (1) and (2) $2 d_{R}(a) b a+d_{R}(b) a^{2}=d_{R}(a)(a b+b a)+d_{R}(b) a^{2}$

$$
\begin{aligned}
& d_{R}(a) b a=d_{R}(a) a b \\
& \quad d_{R}(a)[a, b]=0 \quad \forall a, b \in S
\end{aligned}
$$

Replacing $b$ by $c b$, and using (3) we get

$$
d_{R}(a) c[a, b]=0 \quad \forall a, b, c \in S \Rightarrow d_{R}(a) S[a, b]=0 \quad \forall a, b \in S .
$$

Since $d_{R} \neq 0$, we get $[a, b]=0 \forall a, b \in S$, thus proving that $S$ is commutative.

Analogously we can prove the following.

Theorem 3.11 Let $S$ be an additively commutative and cancellative semiring. Let $X$ be a left $S-$ module. Let $d_{L}: S \rightarrow X$ be a non zero left derivation. Then $S$ is commutative.

\section{References}

[1] Bresar. M and Vukman.J: On left derivations and related mappings,Proc. Amer. Math. Soc., Vol 110, No: 1 Sep (1990), 7-16.

[2] Chandramouleeswaran.M and Thiruveni.V : On derivations of semirings Advances in Algebras Vol (3) (2010), 123 - 131.

[3] Jonathan S.Golan: Semirings and their Applications Kluwer Academic Press 1969

[4] Posner. E.C: Derivations in prime rings, Proc. Amer. Math. Soc., Vol 8 (1957), 1093 - 1100.

[5] Vandiver. H.S: Note on a simple type of algebra in which the cancellation law of addition does not hold, Bull. Amer. Math. Soc., Vol 40, (1934), 916-920.

\section{Received: August 1, 2013}

